\title{
A Firefly Optimized Multi Adaptive Parallel Neuro Fuzzy Inference System
}

\author{
Dr. D. Revathi \\ Assistant Professor, \\ Department of Information Technology, \\ Bharathidasan College of Arts and Science, Erode - 638116.
}

\begin{abstract}
In this research the firefly optimized multi adaptive parallel neuro-fuzzy inference (FOMAPNFI) system introduce for improving the performance of the system. This method is inspired from fireflies which produce short flashes as a protective system and to attract mates or prey. The rate and rhythm of the flashes, as well as the time interval between them attract two sexes toward each other. The intensity of light is decreased following an increase in distance from light source. The transmitted light is used as the formulated objective function. Three important properties of FA algorithm are: 1) Firefly is brighter and more attractive when it moves accidentally and all fireflies are unisexual; 2) Attractiveness of firefly is proportional to the brightness and distance from it, and decrease in light intensity is calculated by light absorption coefficient. Brightness of firefly is determined by the objective function value. 3) The distance between each firefly is obtained through objective function.

Then consider the Multi adaptive parallel neurofuzzy inference system along with additional optimized parameters by using firefly algorithm. This research is used for removing the impulse noise efficiently rather than other methods. Each member of a group of fireflies moved toward a point where their best experience had occurred. By using this approach the best and multiple membership parameters are selected. Hence it is used for which improving the performance of denoising superior.
\end{abstract}

Keywords: Firefly algorithm, Fuzzy rules, Neural Network, Membership function

\section{INTRODUCTION}

Over the past decades, different image denoising approaches have been developed for removing the impulse noise. The process of removing the noise from the images is mostly required for enhancing the restored images in image processing. Due to the noise removal operator, such approaches are having high computation complexity and difficult to preserve the useful information during noise removal process. In previous researches, MAPNFIS is proposed for removing the processing speed of denoising process with the multiple parameters. In this approach, Multi-AFIS is hybridized with the NN for improving the performance speed of the denoising process and the selection of fuzzy membership function is done in adaptive manner. The computation of weighting factors of the rules is used for selecting the membership function adaptively. However, the performance of denoising is still less due to high noise rate and selection of optimal membership function.
Hence in this research phase, a Firefly Optimized Multi Adaptive Parallel Neuro-Fuzzy Inference System (FOMAPNFIS) is proposed. In this method, firefly algorithm is introduced for selecting the optimized membership function of fuzzy rules which is utilized for denoising process. By using this approach, the best and multiple membership parameters are selected. Hence it is used for which improving the performance of denoising superior. Finally, the experimental results show that the proposed FOMAPNFI system achieves better performance than the other image denoising approaches.

\section{RELATED WORK}

A new method of noise removal ${ }^{5}$ was proposed for the images corrupted by impulse noise. The main aim of this method was combining median and adaptive median filter for achieving better outcomes in terms of computation time and visual quality. In this method, the corrupted pixels were replaced by using a median filter. Otherwise, they were estimated by their neighbour's values. However, PSNR value was less.

Medical image denoising method ${ }^{4}$ was proposed by using convolutional denoising auto-encoders. In this approach, denoising auto-encoder was constructed by using convolutional layers for removing the noise from the medical images. However, the performance of denoising was less when training sample size was increased.

Fuzzy based salt-and-pepper noise removal ${ }^{9}$ was proposed by using adaptive switching median filter. In this method, two phases were considered such as detection and filtering. In the detection phase, neighbourhood mapping based algorithm was used for detecting the corrupted pixels. In the filtering phase, the corrupted pixels were filtered by using fuzzy membership function so the uncorrupted pixels were retained. This algorithm was used for variable impulse noise and there was no requirement of threshold for detection process. However in this method, the local information in the image was not estimated.

An efficient method of image denoising ${ }^{3}$ was proposed by using hybrid filter approach. The major objective of this approach was estimating the uncorrupted image from the distorted or noisy image. This approach was performed based on wiener filter and Pseudo inverse filter. However, the time complexity and complexity of the algorithm were high. 


\section{FIREFLY OPTIMIZED MULTI ADAPTIVE}

\section{PARALLEL NEURO-FUZZY INFERENCE SYSTEM}

In this section, the proposed FOMAPNFIS for removing the impulse noise in the images is explained in brief. This approach is used for optimizing the membership functions utilized in MAPNFIS for obtaining the best outcome by including the firefly optimization algorithm. Initially, MAPNFIS is briefly explained in section 5.2. Here, how firefly optimization algorithm is used for training the MAPNFIS for obtaining the best fuzzy membership functions with minimal error function is explained in below.

\subsection{Firefly Optimization Algorithm}

Basically, MAPNFIS has 5 layers and each layer has its own membership function. ${ }^{1}$ In each layer, the membership function is optimized by firefly algorithm. ${ }^{10}$ Generally, firefly algorithm follows three rules which are given in below.

- All fireflies are unisex. Each firefly can attract the other fireflies regardless of their sex.

- Attractiveness is proportional to the brightness and inversely to the distance. It refers a firefly that has less brightness will move towards the brighter one and if there is no firefly in that region, it will move randomly.

- The brightness or light intensity of a firefly is determined by the objective function which is to be optimized.

Initially, the fuzzy rules are generated according to the three inputs such as $X_{1}, X_{2}, X_{3}$ and the generated fuzzy rules are given as input to the first layer of MAPNFIS. Then, the firefly algorithm is applied for training the fuzzy rules for optimizing the fuzzy rules to get a specific range of solution based on the set of constraints and the fitness function. In this research, PSNR, MSE and FCR are used as the fitness function for evaluating the quality of MAPNFIS. The overall process of this approach is shown in Figure

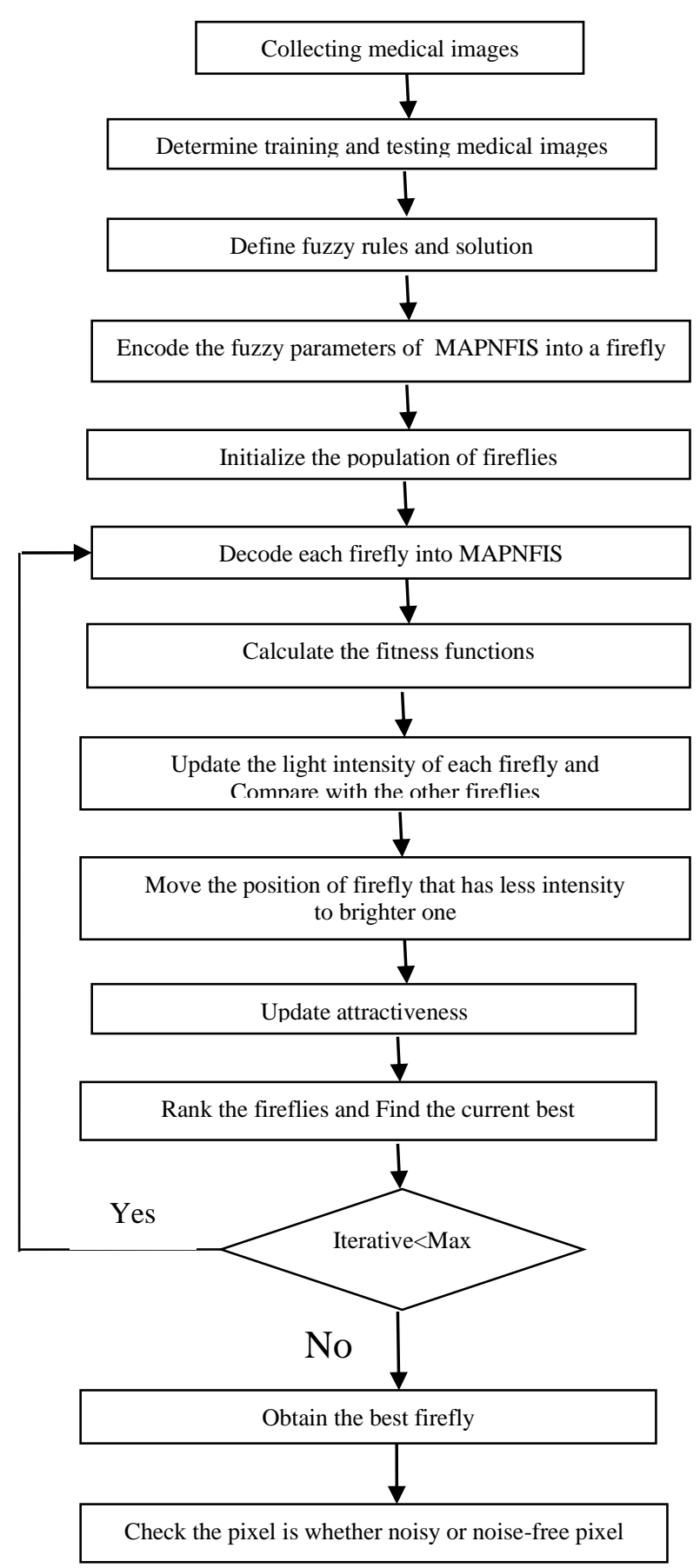

Fig.1.1 Overall Flow Diagram of FOMAPNFIS

Consider the set of fireflies which is represented as a population. Each input of the second layer is represented by a firefly. Each firefly has two parts such as set of antecedent parameters and set of consequent parameters. The parameters which are required for adjusting MAPNFIS are coded into the individual real number code chain i.e., the network parameters like $m_{i j}, \sigma_{i j}, c_{i 1}, c_{i 2}, c_{i 3}$ and $c_{i 4}$ are integrated together. Based on the population of fireflies, a group of fireflies will be generated randomly and each firefly is mapped into MAPNFIS's parameter set. After that, the fitness value of each firefly is calculated. The light 
intensity of each firefly is computed by using the fitness function.

$$
\begin{aligned}
f_{1}\left(x_{i}\right)=M S E & =\frac{1}{L C} \sum_{l=1}^{L} \sum_{c=1}^{C}(s[l, c]-\gamma[l, c])^{2} \\
f_{2}\left(x_{i}\right)=F C R & =\frac{N_{F}}{N_{T}} \\
& \times 100 \\
f_{3}\left(x_{i}\right)= & P S N R=20 \times \log _{10}\left(\frac{255}{\sqrt{M S E}}\right)
\end{aligned}
$$

Once the light intensity of each firefly is computed, the attractiveness between fireflies is compared based on the computed light intensity and move fireflies that have less brightness to the highest brighter firefly. The attractiveness is varied according to the distance between the fireflies. Moreover, the computation of fitness function is repeated until the system fitness meets either the fitness threshold or the iterative number is larger than the maximum allowable iterative number. Then, the process is terminated and the fireflies are sorted based on their attractiveness. Finally, the output is the position and the fitness value of the best firefly. Once the training process is completed, the testing images are analyzed for classifying the image pixels whether it is noisy or noise-free pixel effectively in adaptive manner and using median filter.

Algorithm: FOMAPNFIS based image denoising approach Input: Training images

Output: Classification of noisy and noise-free pixels

1. The nearest pixels of the center pixel are given to the sub detectors and noise filter.

2. Convert the scalar input values into fuzzy numbers and generate fuzzy rules.

3. The fuzzy rules are scattered to the nodes for parallel processing and compute the type-1 interval fuzzy set.

4. Initialize the number of fireflies for each fuzzy set.

5. Define objective functions $f_{1}\left(x_{i}\right), f_{2}\left(x_{i}\right)$ and $f_{3}\left(x_{i}\right)$.

6. Define operating parameters $m_{i j}, \sigma_{i j}, c_{i 1}, c_{i 2}, c_{i 3}$ and $c_{i 4}$ for each firefly.

7. Compute the light intensity $I_{i}$ at $x_{i}$ based on the objective functions.

8. Define the absorption coefficient $\gamma$ of each firefly.

9. while $(t<$ MaxGeneration $)$

10. for $i=1: n$ (all $n$ fireflies)

11. for $j=1: n$ ( $n$ fireflies)

12. if $\left(I_{j}>I_{i}\right)$

13. Move firefly $i$ towards $j$.

14. End if

15. Update attractiveness.

16. Evaluate the new solutions and update light intensity.

17. End for $j$

18. End for $i$

19. Rank fireflies and find the current best.

20. End while

21. Obtain the best solution.
22. Classify the pixel as whether noisy or noise-free pixel.

\section{EXPERIMENTAL RESULTS}

In this section, the performance effectiveness of the proposed Firefly Optimized Multi Adaptive Parallel Neuro-Fuzzy Inference System (FOMAPNFIS) is evaluated and compared with the Multiple Adaptive Parallel Neuro Fuzzy Inference System (MAPNFIS) based Impulse detector in terms of mean squared error, false classification ratio and processing time, peak signal-tonoise ratio. MATLAB simulation environment is used to prove the improvement and the successful execution of the environment in case of presence of more noisy images. In the experiments, the noisy analysis medical pictures are non inheritable by contaminating a given testing picture with an impulse noise of given noise density. Numerous noise density values are used such are $25 \%, 50 \%$ and $75 \%$ which indicates the low, medium and high noise densities, correspondingly.

\subsection{Mean Squared Error}

Mean Squared Error (MSE) is defined as follows:

$$
M S E=\frac{1}{L C} \sum_{l=1}^{L} \sum_{c=1}^{C}(s[l, c]-\gamma[l, c])^{2}
$$

In above equation, $s[l, c]$ and $\gamma[l, c]$ denotes the luminance value of the pixel at line $l$ and column $c$ of one of the three color bands of the original and the restored versions of a corrupted test image correspondingly. The MSE is valid for the gray-scale images. But the testing images are color images. So, the MSE computation is performed for three times, one for each color bands and the three resulting MSE values are averaged to acquire the particular MSE value for the image.

The MSE comparison for the existing and proposed system is shown in Table 1.1. If the noise density is $75 \%$, the MSE is 46 in the existing MAPNFIS method and 41 in the proposed method.

Table.1.1 Comparison of MSE

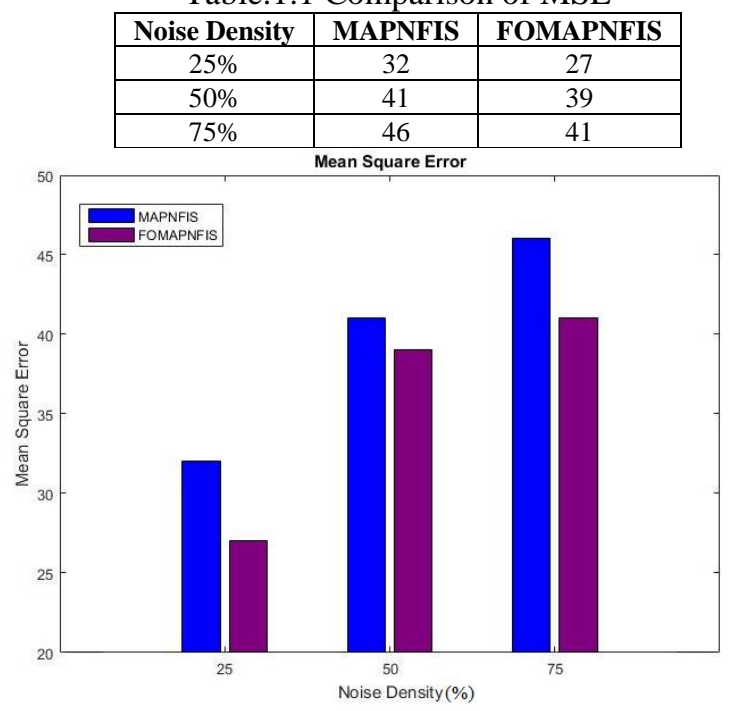

Fig.1.2 Comparison of MSE 
Figure 1.2 shows that the comparison of MSE for existing and proposed system. In X-axis, the noise densities are taken in \%. In y-axis, MSE values are considered. If the noise density is $25 \%$, then the MSE of proposed FOMAPNFIS and MAPNFIS are 32 and 27 respectively. When the noise density is $50 \%$, the corresponding MSE value of proposed FOMAPNFIS and MAPNFIS are 41 and 39 respectively. Also, if the noise density is $75 \%$, then the MSE value of proposed FOMAPNFIS and MAPNFIS are 46 and 41 correspondingly. From the analysis, it is observed that the proposed FOMAPNFIS has less mean square error value compared with the existing method.

\subsection{False Classification Ratio}

False classification ratio is defined as follows:

$$
F C R=\frac{N_{F}}{N_{T}} \times 100
$$

Where $N_{F}$ denotes the falsely classified pixels of the input image and $N_{T}$ represents the total number of pixels.

The FCR comparison for the existing and proposed system is shown in Table 4.2. If the noise density is $75 \%$, the FCR is $3.6 \%$ in the existing FOPFIS method and $2.7 \%$ in the proposed method.

Table.1.2 Comparison of FCR (\%)

\begin{tabular}{|c|c|c|}
\hline Noise Density & MAPNFIS & FOMAPNFIS \\
\hline $25 \%$ & 1.5 & 1 \\
\hline $50 \%$ & 2.8 & 2.1 \\
\hline $75 \%$ & 3.6 & 2.7 \\
\hline
\end{tabular}

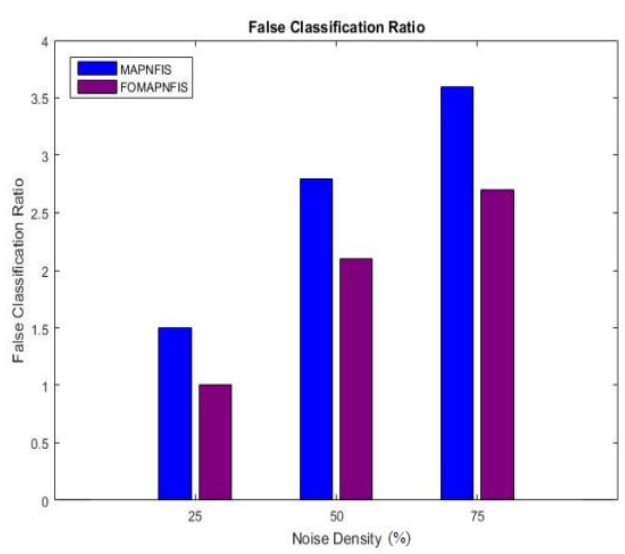

Fig.1.3 Comparison of FCR

Figure 1.3 shows that the comparison of FCR for existing and proposed system. In $\mathrm{x}$-axis, the noise densities are taken in \%. In y-axis, FCR values are considered in \%. If the noise density is $25 \%$, then the FCR of proposed FOMAPNFIS and MAPNFIS are $1.5 \%$ and $1 \%$ respectively. When the noise density is $50 \%$, the corresponding FCR value of proposed FOMAPNFIS and MAPNFIS are $2.8 \%$ and $2.1 \%$ respectively. Also, if the noise density is $75 \%$, then the FCR value of proposed FOMAPNFIS and MAPNFIS are $3.6 \%$ and $2.7 \%$ correspondingly. From the analysis, it is observed that the proposed FOMAPNFIS has less false classification ratio compared with the existing method.

\subsection{Computation Time}

Computation time is defined as the time taken for processing the images and filters the noise.

The comparison of computation time for the existing and proposed system is shown in Table 1.3. If the noise density is $75 \%$, the computation time is $1.91 \mathrm{~ms}$ in the existing MAPNFIS method and $1.4 \mathrm{~ms}$ in the proposed method.

Table.1.3 Comparison of Computation Time (ms)

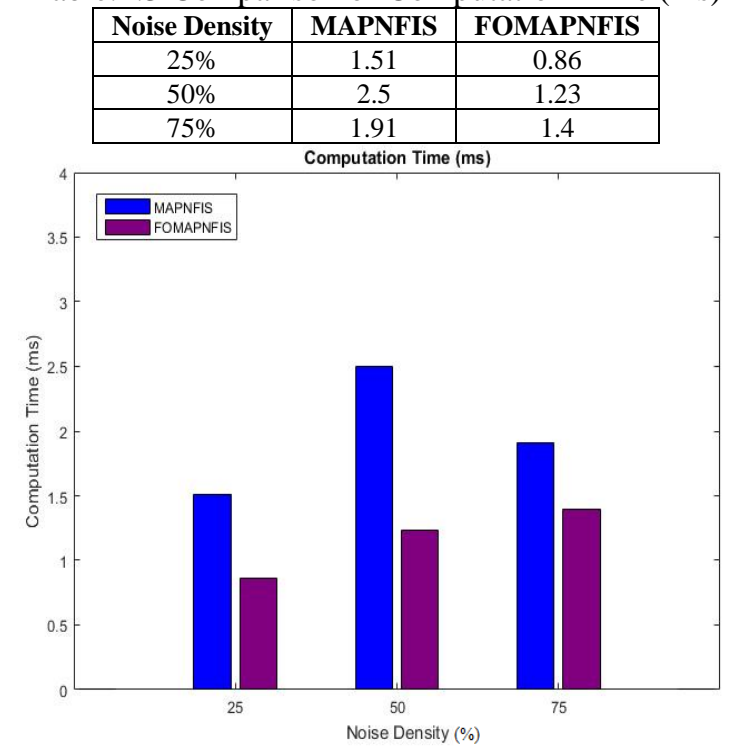

Fig.1.4 Comparison of Computation Time

Figure 1.4 shows that the comparison of computation time for existing and proposed system. In X-axis, the noise densities are taken in \%. In y-axis, computation time values are considered in milliseconds. If the noise density is $25 \%$, then the computation time of proposed FOMAPNFIS and MAPNFIS are $1.51 \mathrm{~ms}$ and $0.86 \mathrm{~ms}$ respectively. For the noise density is $50 \%$, the corresponding computation time of proposed FOMAPNFIS and MAPNFIS are $2.5 \mathrm{~ms}$ and $1.23 \mathrm{~ms}$ respectively. Also, if the noise density is $75 \%$, then the computation time of proposed FOMAPNFIS and MAPNFIS are $1.91 \mathrm{~ms}$ and $1.4 \mathrm{~ms}$ correspondingly. From the analysis, it is observed that the proposed FOMAPNFIS has less computation time compared with the existing method.

\subsection{Peak Signal-to-Noise Ratio}

Peak signal-to-noise ratio (PSNR) is used to represent the index of media quality analysis and substitutes the average MSE of frames in the PSNR computing equation to obtain the PSNR value of the media segment and it is expressed as follows:

$$
\text { PSNR }=20 \times \log _{10}\left(\frac{255}{\sqrt{\mathrm{MSE}}}\right)
$$

The PSNR comparison for the existing and proposed system is shown in Table 1.4. If the noise density is $75 \%$, the PSNR is $90 \%$ in the existing MAPNFIS method and $94 \%$ in the proposed method. 


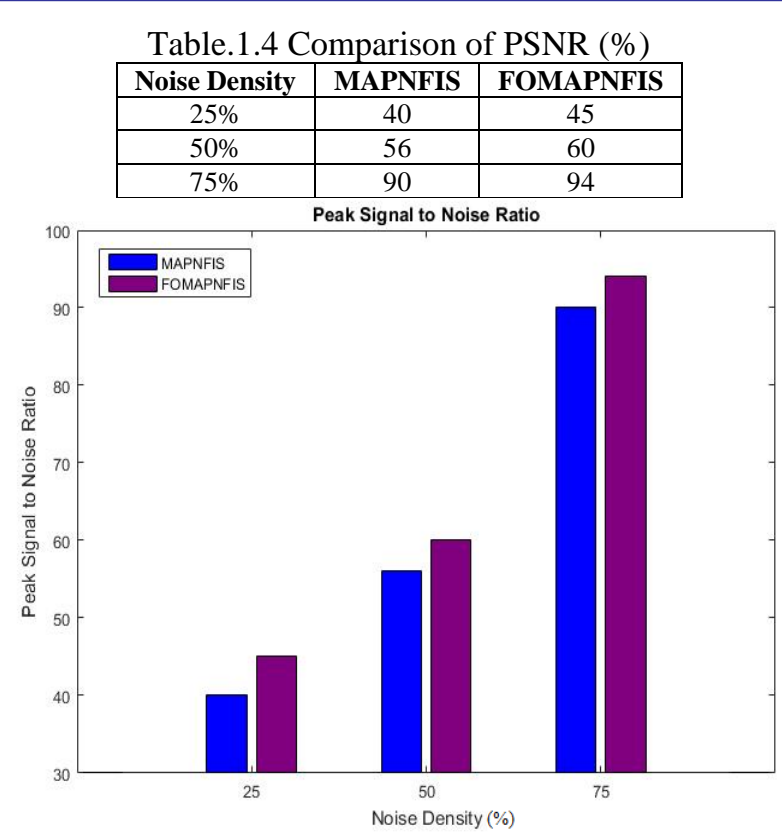

Fig.1.5 Comparison of PSNR

Figure 1.5 shows that the comparison of PSNR for existing and proposed system. In $\mathrm{x}$-axis, the noise densities are taken in \%. In y-axis, PSNR values are considered in terms of $\%$. If the noise density is $25 \%$, then the PSNR of proposed FOMAPNFIS and MAPNFIS are $40 \%$ and $45 \%$ respectively. When the noise density is $50 \%$, the corresponding PSNR value of proposed FOMAPNFIS and MAPNFIS are $56 \%$ and $60 \%$ respectively. Also, if the noise density is $75 \%$, then the PSNR value of proposed FOMAPNFIS and MAPNFIS are $90 \%$ and $94 \%$ correspondingly. From the analysis, it is observed that the proposed FOMAPNFIS has less peak signal-to-noise ratio compared with the existing method.

\section{CONCLUSION}

In this paper, Firefly Optimized Multi Adaptive Parallel Neuro-Fuzzy Inference System (FOMAPNFIS) is introduced for enhancing the noise removing process in image processing. In this method, firefly optimization algorithm is applied for further improving the multi adaptive Neuro-fuzzy system with consideration of multiple parameters. The major objective of this method is optimizing the fuzzy parameters for improving the quality of the image denoising performance efficiently. It optimizes the selection of fuzzy membership functions for deciding the noisy and noise-free pixels in the images effectively. Finally, the experimental results prove that the proposed FOMAPNFIS has better performance compared to the other image denoising approaches in terms of MSE, PSNR, MAE and computation time.

\section{REFERENCES}

1. Benmiloud, T. (2010, June). Multi-output adaptive Neuro-fuzzy inference system. In WSEAS international conference on neural networks (Vol. 11, pp. 94-98).

2. Choubey, A., Sinha, G. R., \& Choubey, S. (2011, April). A hybrid filtering technique in medical image denoising: Blending of neural network and fuzzy inference. In Electronics Computer Technology (ICECT), 2011 3rd International Conference on (Vol. 1, pp. 170-177). IEEE.
3. Detani, D., Upadhyay, A., \& Saxena, M. An Efficient Method of Image Denoising Using Hybrid Filter Approach. IOSR Journal of Electronics and Communication Engineering, 9(4), 79-84.

4. Gondara, L. (2016, December). Medical image denoising using convolutional denoising autoencoders. In Data Mining Workshops (ICDMW), 2016 IEEE 16th International Conference on (pp. 241246). IEEE.

5. Jourabloo, A., Feghahati, A. H., \& Jamzad, M. (2012). New algorithms for recovering highly corrupted images with impulse noise. Scientia Iranica, 19(6), 1738-1745.

6. Khalifa, A. B., \& Frigui, H. (2015, August). MI-ANFIS: A multiple instance adaptive neuro-fuzzy inference system. In Fuzzy Systems (FUZZ-IEEE), 2015 IEEE International Conference on (pp. 1-8). IEEE.

7. Muosa, A. H., \& Hamad, A. M. (2015). Mixed noise denoising using Neuro fuzzy and memetic algorithm. International Journal of Research in Computer Applications and Robotics, 3(12), 16-23.

8. Shaabani, M. E., Banirostam, T., \& Hedayati, A. (2016). Implementation of Neuro fuzzy system for diagnosis of multiple sclerosis. International Journal of Computer Science and Network, 5(1), 157-164.

9. Thirilogasundari, V., \& Janet, S. A. (2012). Fuzzy based salt and pepper noise removal using adaptive switching median filter. Procedia Engineering, 38, 2858-2865.

10. Nhu, H. N., Nitsuwat, S., \& Sodanil, M. (2013, September). Prediction of stock price using an adaptive Neuro-Fuzzy Inference System trained by Firefly Algorithm. In Computer Science and Engineering Conference (ICSEC), 2013 International (pp. 302-307). IEEE.

11. Yang, X. S., \& He, X. (2013). Firefly algorithm: recent advances and applications. International Journal of Swarm Intelligence, $1(1), 36-50$.

12. Yuksel, M. E., \& Basturk, A. (2012). Application of type-2 fuzzy logic filtering to reduce noise in color images. IEEE Computational intelligence magazine, 7(3), 25-35. 